ESAIM: M2AN 49 (2015) 1659-1670

DOI: $10.1051 / \mathrm{m} 2 \mathrm{an} / 2015026$
ESAIM: Mathematical Modelling and Numerical Analysis

www.esaim-m2an.org

\title{
OPTIMAL PITS AND OPTIMAL TRANSPORTATION
}

\author{
IVAR EKELAND ${ }^{1}$ AND MAURICE QUEYRANNE ${ }^{2,3}$
}

\begin{abstract}
In open pit mining, one must dig a pit, that is, excavate the upper layers of ground before reaching the ore. The walls of the pit must satisfy some geomechanical constraints, in order not to collapse. The question then arises how to mine the ore optimally, that is, how to find the optimal pit. We set up the problem in a continuous (as opposed to discrete) framework, and we show, under weak assumptions, the existence of an optimum pit. For this, we formulate an optimal transportation problem, where the criterion is lower semi-continuous and is allowed to take the value $+\infty$. We show that this transportation problem is a strong dual to the optimum pit problem, and also yields optimality (complementarity slackness) conditions.
\end{abstract}

Mathematics Subject Classification. 37A05, 49J20, 49J45, 90C26, 90C35, 90C48.

Received April 2, 2015.

Published online November 5, 2015.

\section{INTRODUCTION}

In open pit mining, one tries to extract profitable ore, but for this one has to excavate less profitable layers of soil or rock above it. One thereby digs a hole, the pit, deep enough to reach the underground ore. The walls cannot be too steep, otherwise the hole will cave in; therefore one has to impose constraints on the slopes. These constraints depend on the nature of the rock, and may vary with depth and location. In any case, the deeper the ore, the wider the pit, and the costlier it is to reach it. Certain parts will simply be too costly to reach, so the question arises: how to determine a most profitable pit? Note that this question encompasses several others, such as: how deep to dig? what is the shape of the hole, taking into account the slope constraints? which parts of the ore can be most profitably exploited, and which parts are too expensive to reach? which parts will be processed, and which ones will go to waste ("cut-off grade" decisions)? what is the net economic value of the deposit? what will be the return on investment from this mining project?

Since the 1960s, the standard approach has been to discretize this "ultimate pit limits" problem and to solve it by linear programming and related network flow methods; see Section 2 for details and some references. However, this is by nature a problem in continuous space: the ore density and other rock properties tend to

\footnotetext{
Keywords and phrases. Optimal transportation, optimal pit mine design, Kantorovich duality.

1 CEREMADE, Université Paris-Dauphine, Place du Maréchal De Lattre De Tassigny, 75775 Paris, France. ekeland@math.ubc.ca

2 CORE, Université Catholique de Louvain, Voie du Roman Pays 34, 1348 Louvain-la-Neuve, cedex 16, Belgium, France.

3 Sauder School of Business, University of British Columbia, 2053 Main Mall, Vancouver, BC V6T 1Z2, Canada.
} 
vary continuously underground, and their distributions are estimated ("smoothed") from sample (drill hole) data and other geological information.

There were some attempts to formulate and analyze the ultimate pit limits problem in continuous space, also reviewed in Section 2 below. In the mid-1970s Matheron [12,13] proposed a general formulation of the problem, that encompasses both continuous and discretized approaches; we review this formulation in Section 3 and actually use it later in this paper. Nearly forty years later, Alvarez et al. [1] formulate a constrained optimization (calculus of variations) problem in functional space to determine the optimum depth $\varphi(y)$ at which to dig under every point $y$ on the ground surface, subject to bounds on the derivative of $\varphi$ to model the wall slope constraints. Although both Matheron and Alvarez et al. prove, under mild technical conditions, the existence of optimum pits, their approaches suffer from the lack of convexity in their optimization models and the resulting difficulties of determining and recognizing a global optimum in the presence of (potentially many) local optima.

In this paper, we develop yet another approach, which is to determine an optimum pit from an optimum dual solution to a particular transportation problem. Recall (see, e.g., [19]) that optimal transportation is concerned with transporting mass between origins and destinations at minimum total cost, so its relevance to mining (optimally extracting material from the ground) should come as no surprise. In present work, however, the relationship is not so obvious, as we consider the "transportation", or rather allocation, of profits, and not of rock or ore. The underlying intuition is that in any profitable pit, there are unprofitable parts, with no commercial interest, but which must be cleared out to reach the profitable ones. The revenue from the ore must pay for all the excavation and processing, and still leave a profit. Thus, extending to continuous space earlier discrete models of Johnson [9] and Huttagosol and Cameron [8], we consider the use of profitable parts of the pit to pay for the unprofitable parts which extraction they require. For this, we set up an optimal transportation model where the "transportation costs" are used in part to model the geomechanical slope constraints, and take the value $+\infty$ in some components. The detailed transportation model and its Kantorovich dual are defined in Sections 4 and 5. We use special arguments in Section 6 to deal with the particular cost function and establish that the dual problem admits an optimum solution. Finally, in Section 7 we show that there is an optimum dual solution which actually defines an optimum pit. Thus we obtain a convex (actually linear) programming formulation of the continuous space open pit mine problem, of which the transportation problem is a strong dual. This strong dual pair of infinite dimensional and specially structured linear programming problems also yields a characterization of optimal pits through complementarity slackness conditions.

\section{RELATED WORK}

As mentioned in the Introduction, the standard approach to open pit mine planning and scheduling has, since the 1960s, been to discretize these problems and to solve the resulting approximations by linear or integer programming methods. This leads to highly structured, finite-dimensional linear optimization problems of very large size, typically with hundreds of thousands to several million variables and constraints (see, e.g., [5]), for which specialized algorithms, often based on network flow models $([2,9,16])$ have been developed; see also $[1,15]$ for further details and references. However, the discretization of the volume of interest (ore body) into threedimensional blocks with vertical sides, while appropriate for many aspects of mining operations, only produces a rough approximation of the slope restrictions (see, e.g., the 5:1 and 9:1 block patterns in Fig. 2 in [15], or the discussion of "Assumption 5", pp. 27-33 in [9]).

In unpublished technical notes [12,13], Georges Matheron proposed a general formulation of the ultimate pit limits problem, that encompasses both continuous space and discretized approaches. Under very mild mathematical assumptions, Matheron proves the existence of an optimum pit (an issue which, of course, does not arise with finite discretized models), as well as general lattice properties of the set of all optimum pits (stability under countable unions and intersections; see Cor. 7.4 below and the discussion preceding it). Matheron's primary interest is in parametric properties of optimum pits relative to ore grade, and he characterizes optimum pits in 
these terms. However his results do not appear to have useful algorithmic implications for finding an optimum pit, and the various heuristic methods that he discusses may all fail to produce near-optimal pits.

More recently, Morales [14] considered underground mine design in continuous space, while Gúzman [7] proposed shape and topological optimization methods for open pit mine design.

Alvarez et al. [1] present a different continuous space approach to the ultimate pit limit problem. They model the shape of the pit as the graph of a pit depth function $\varphi: A \rightarrow R$, where $A \subset \mathbb{R}^{2}$ is the "claim", i.e., the surface area under which the pit will be dug, and $\varphi(y)$ is the depth at which the pit will extend under point $y \in A$. The geomechanical requirements on the pit slopes are formulated by requiring that at every point $y \in A$ the local Lipschitz constant of $\varphi$ does not exceed a given upper bound $\omega(y, \varphi(y))$. Thus, this constraint prescribes the maximal stable local slope and may vary on with location $y \in A$ and depth $z=\varphi(y)$, depending on the local geotechnical properties of the material. This leads to a constrained optimization (calculus of variations) problem in functional space, for which they prove existence (an issue that was not considered by Morales or Gúzman) and some qualitative properties of optimum solutions, some of which related to those in Matheron's work $^{4}$. A drawback of this Lipschitz-constrained depth function approach, however, is the lack of convexity in the resulting optimization problem, with the possible existence of local optima and the difficulty of recognizing or characterizing a global optimum (see also $[6,17]$ for attempts to address such issues). Another limitation is the isotropy of the Lipschitz local slope constraint: geological features such as tilted rock layers, folding, faults and other rock structures may cause the wall stability to depend on the wall orientation, thus resulting in slope constraints that are anisotropic (see, e.g., [11] and the extensive discussion in Sect. 2.3 in [10]).

\section{THE MODEL}

We are given a compact subset $E \subset \mathbb{R}^{3}$, representing the domain to be mined. (For example, we may think of $E$ as having the special form $E=A \times\left[h_{1}, h_{2}\right]$, where $A \subset \mathbb{R}^{2}$ is the claim, and $\left[h_{1}, h_{2}\right]$ is the elevation or depth range.) As in Matheron [12], we represent the geomechanical constraints defining feasible pits by assuming that we are given a (point-to-set) map $\Gamma: E \rightarrow E$ where for every $x \in E, \Gamma(x)$ is the set of all points that it is necessary and sufficient to extract in order to extract $x$ itself. In mining terms, we may think of $\Gamma(x)$ as the "cone" which has to be excavated in order to mine $x$. We assume that $\Gamma$ has a closed graph and satisfies:

$$
\begin{array}{ll}
\text { (reflexivity) } & x \in \Gamma(x) \\
\text { (transitivity) } & {\left[x^{\prime} \in \Gamma(x) \text { and } x^{\prime \prime} \in \Gamma\left(x^{\prime}\right)\right] \Longrightarrow x^{\prime \prime} \in \Gamma(x)}
\end{array}
$$

These last two properties imply that $\Gamma$ induces a preorder ${ }^{5} \succsim_{\Gamma}$ on $E$, defined by

$$
x^{\prime} \succsim_{\Gamma} x \quad \text { if and only if } \quad x^{\prime} \in \Gamma(x) .
$$

Thus $x^{\prime} \succsim_{\Gamma} x$ means that one must extract $x^{\prime}$ in order to reach $x$. Since

$$
\Gamma(x)=\left\{x^{\prime} \in E: x^{\prime} \succsim_{\Gamma} x\right\},
$$

it is equivalent to specify the map $\Gamma$ or the preorder $\succsim_{\Gamma}$.

Definition 3.1. A pit is a subset $F \subseteq E$ which is Lebesgue measurable and stable for the preorder, i.e., satisfies

$$
\left[x \in F \text { and } x^{\prime} \succsim_{\Gamma} x\right] \Longrightarrow x^{\prime} \in F \text {. }
$$

\footnotetext{
${ }^{4}$ Alvarez et al. [1] also consider capacitated and dynamic versions of the problem, that is, open pit mine scheduling problems, which are beyond the scope of the present work.

${ }^{5}$ In most cases $x$ is at a greater depth than every other point in $\Gamma(x)$. Then $\succsim_{\Gamma}$ is also antisymmetric, and thus a partial order. As noted by Matheron [12], however, this assumption, while natural in the mining context, is not actually needed in this work.
} 
To reach an underground region $F \subset E$, one must excavate the whole pit, up to ground level. Thus, letting

$$
\Gamma(F):=\cup_{x \in F} \Gamma(x)
$$

and $\mathcal{L}(E)$ denote the family of all Lebesgue measurable closed subsets $F \subseteq E$, we have that

$$
F \in \mathcal{L}(E) \text { is a pit if and only if } \Gamma(F)=F \text {. }
$$

Finally, we are given a continuous function $g: E \rightarrow \mathbb{R}$ representing net profit, namely, $g(x) \mathrm{d} x$ is the net profit directly resulting from the extraction and processing of the volume element $\mathrm{d} x=\mathrm{d} x_{1} \mathrm{~d} x_{2} \mathrm{~d} x_{3}$ at $x$ after all $x^{\prime} \succsim_{\Gamma} x$ have already been extracted. The regions where $g>0$ are those of profitable ore (the higher $g$, the richer the ore); the regions where $g \leq 0$ represent waste, which may have to be extracted to reach profitable ore. We assume that the net profit function $g$ satisfies

$$
\int_{E} \max \{0, g(x)\} \mathrm{d} x>0,
$$

for otherwise there is no profitable ore, and thus no hope of making a profit. If we want to extract the ore from a region $F \subseteq\{g>0\}$, one has to excavate all the ground above it, that is, the whole pit $\Gamma(F)$, and the corresponding profit is

$$
\int_{\Gamma(F)} g(z) \mathrm{d} z .
$$

To summarize, the data are $E, \Gamma$ and $g$. The family of all pits will be denoted by $\mathcal{S}(E)$ : for all $f \in \mathcal{L}(E)$

$$
F \in \mathcal{S}(E) \Longleftrightarrow F=\Gamma(F)
$$

We are looking for a pit that maximizes profit, that is, we are aiming to solve the optimization problem:

$$
\begin{aligned}
& \max \int_{F} g(z) \mathrm{d} z \\
& \text { s.t. } F \in \mathcal{S}(E)
\end{aligned}
$$

\section{An optimal tRANSPORTATION PROBLEM}

As outlined in the Introduction, we define an optimal transportation problem to allocate as much a possible of the profits from the profitable parts of the ore body to pay for the unprofitable parts they require to extract. This should leave unallocated profits, which we allocate to a sink $\omega$; thus the amount put into $\omega$ is meant to represent the pit net profit. There may also be unprofitable parts which are not fully paid for, meaning that they should be left unexcavated; to account for these, we introduce a source $\alpha$, which we connect to all unprofitable parts. As a result, we define the following compact subsets of $E$ :

$$
E^{+}:=\overline{\{g(x)>0\}} \text { and } E^{-}:=\overline{\{g(x)<0\}} .
$$

Add the source $\alpha$ and the sink $\omega$ and let:

$$
X:=E^{+} \cup\{\alpha\} \quad \text { and } \quad Y:=E^{-} \cup\{\omega\} .
$$

Both $X$ and $Y$ are compact sets. We endow them with the non-negative measures $\mu$ and $\nu$ defined by:

$$
\begin{array}{ll}
\mu(\{\alpha\})=\int_{E^{-}}|g(z)| \mathrm{d} z, & \left.\mu\right|_{E^{+}}=g(z) \mathrm{d} z \\
\nu(\{\omega\})=\int_{E^{+}} g(z) \mathrm{d} z, & \left.\nu\right|_{E^{-}}=|g(z)| \mathrm{d} z .
\end{array}
$$

Thus $\nu(\{\omega\})$ is the total profit of all profitable parts (an upper bound on the net profit of any pit), and $\mu(\{\alpha\})$ is the total cost (negative profit) of all unprofitable parts. Note that $\mu\left(E^{+}\right)>0, \mu\left(E^{+} \cap E^{-}\right)=\nu\left(E^{+} \cap E^{-}\right)=0$, and $\mu(X)=\nu(Y)$ represents the total "mass" (positive profits and costs) to be "transported" (allocated). 
Profit allocations are allowed from every profitable $x \in E^{+}$to every $y \in \Gamma(x) \cap E^{-}$, as well as between the source $\alpha$ and all $y \in E^{-}$(unpaid costs); and also between all $x \in E^{-}$and the sink $\omega$ (unallocated, or "excess" profits). We model this using the following "transportation" cost function $c: X \times Y \longrightarrow R$ :

\begin{tabular}{ccc}
\hline \multicolumn{1}{c}{$Y$} & $Y$ & $c(x, y)$ \\
\hline$x \in E^{+}$ & $y \in \Gamma(x)$ & 0 \\
$x \in E^{+}$ & $y \notin \Gamma(x), y \in E^{-}$ & $+\infty$ \\
$x \in E^{+}$ & $y=\omega$ & 1 \\
$x=\alpha$ & $y \in Y$ & 0 \\
\hline
\end{tabular}

The infinite costs represent profit allocations that are not permissible, and the objective of maximizing the total allocated positive profits is represented by the equivalent objective of minimizing the total unallocated profit from $E^{+}$to $\omega$, hence the unit $\operatorname{costs} c(x, \omega)=1$ for all $x \in E^{+}$. (The resulting transportation problem will turn out to be a "dual" of problem $(\mathrm{P})$, which is why we want to minimize total unallocated profits $\left.{ }^{6}\right)$.

Lemma 4.1. $c$ is lower semi-continuous (l.s.c.).

Proof. Let $\left(x_{n}, y_{n}\right) \rightarrow(\bar{x}, \bar{y})$. If $\liminf c\left(x_{n}, y_{n}\right)=+\infty$, there is nothing to prove. If $\liminf c\left(x_{n}, y_{n}\right)<+\infty$, there is a subsequence $n(k)$ such that either

$$
\liminf c\left(x_{n}, y_{n}\right)=c\left(x_{n(k)}, y_{n(k)}\right)=0 \text { for all } n
$$

or

$$
\liminf c\left(x_{n}, y_{n}\right)=c\left(x_{n(k)}, y_{n(k)}\right)=1 \text { for all } n \text {. }
$$

The first case divides into two subcases. Either $x_{n(k)}=\alpha$ for infinitely many $k$, or $x_{n(k)} \in E^{+}$and $y \in \Gamma(x)$ for all $k \geq k_{0}$. In the first subcase, $\bar{x}=\alpha$ and $c(\bar{x}, \bar{y})=0=\liminf c\left(x_{n}, y_{n}\right)$. In the second subcase, since $E^{+}$ is compact, $\bar{x} \in E^{+}$, and since $\Gamma$ has closed graph, $\bar{y} \in \Gamma(\bar{x})$, so that $c(\bar{x}, \bar{y})=0=\liminf c\left(x_{n}, y_{n}\right)$ again.

In the second case, $\bar{x} \in E^{+}$and $\bar{y}=\omega$, so $c(\bar{x}, \bar{y})=1=\liminf c\left(x_{n}, y_{n}\right)$.

Let $\Pi(\mu, \nu)$ denote the set of all positive Radon measures $\pi$ with marginals $\pi_{X}=\mu$ and $\pi_{Y}=\nu$. We consider the optimal transportation problem in Kantorovich form:

$$
\begin{aligned}
& \min \int_{X \times Y} c(x, y) \mathrm{d} \pi \\
& \text { s.t. } \pi \in \Pi(\mu, \nu)
\end{aligned}
$$

Proposition 4.2. Problem $(K)$ has a solution.

Proof. The set of positive Radon measures on the compact space $X \times Y$ is weak-* compact, and the map $\pi \rightarrow \mathrm{E}^{\pi}[c]$ is weak-* 1. s.c.

\section{The Kantorovich DUAL}

Introduce an admissible set $\mathcal{A}$ and a criterion $J$ :

$$
\begin{gathered}
\mathcal{A}:=\left\{(p, q) \in L^{1}(X, \mu) \times L^{1}(Y, \nu) \mid p(x)-q(y) \leq c(x, y) \quad(\mu, \nu) \text {-a.s. }\right\} \\
J(p, q):=\int_{X} p \mathrm{~d} \mu-\int_{Y} q \mathrm{~d} \nu=\int_{E^{+}}(p(z)-q(\omega)) \mathrm{d} \mu-\int_{E^{-}}(q(z)-p(\alpha)) \mathrm{d} \nu
\end{gathered}
$$

\footnotetext{
${ }^{6}$ Note that the problem of maximizing total unallocated profits would be trivial in this setup, for it would be optimum to allocate no profit at all!
} 
These definitions are motivated by the following connections to open pit mine design.

Lemma 5.1. Let $F \in \mathcal{S}(E)$ be a pit. Set $F^{+}:=F \cap E^{+}$and $F^{-}:=F \cap E^{-}$. Define $p_{F}: X \rightarrow R$ and $q_{F}: Y \rightarrow R$ by:

$$
\begin{aligned}
& p_{F}(\alpha)=0, p_{F}(x)= \begin{cases}1 & \text { if } x \in F^{+} \\
0 & \text { otherwise }\end{cases} \\
& q_{F}(\omega)=0, q_{F}(y)= \begin{cases}1 & \text { if } y \in F^{-} \\
0 & \text { otherwise }\end{cases}
\end{aligned}
$$

Then $\left(p_{F}, q_{F}\right)$ is admissible, i.e., in $\mathcal{A}$ and:

$$
J\left(p_{F}, q_{F}\right)=\int_{F} g(z) \mathrm{d} z
$$

is the total net profit associated with the pit $F$.

Proof. Since $F$ is Lebesgue measurable and $X$ and $Y$ are compact we have $p_{F} \in L^{1}(X, \mu)$ and $q_{F} \in L^{1}(Y, \nu)$, and we only need to check that $p_{F}(x)-q_{F}(y) \leq c(x, y)$ for all $x \in X$ and $y \in Y$. If $x=\alpha$, this becomes $q_{F} \geq 0$, which is true. Similarly, if $y=\omega$, we get $p_{F} \leq 1$, which is true as well.

Suppose first $x \in F^{+} \subset E^{+}$, so that $p_{F}(x)=1$. If $y \notin\{\omega\} \cup \Gamma(x)$, we have $c(x, y)=+\infty$, so the relation holds. If $y \in \Gamma(x)$, we must have $y \in F$ because $F$ is a pit, hence stable, so $y \in F \cap E^{-}=F^{-}$and $q_{F}(y)=1$. On the other hand, we have $c(x, y)=0$, so the relation becomes $q_{F}(y) \geq 1$, which is satisfied.

Suppose then $x \notin F^{+}$, so $p_{F}(x)=0$. If $y \notin \Gamma(x)$ then $c(x, y)=+\infty$, and the relation holds. If $y \in \Gamma(x)$, then $c(x, y)=0$, and the relation becomes $q_{F}(y) \geq 0$, which is always true.

As for the last equality, we simply substitute into (5.2), getting:

$$
\begin{aligned}
\int_{X} p_{F} \mathrm{~d} \mu-\int_{Y} q_{F} \mathrm{~d} \nu & =\int_{X}(p(z)-q(\omega)) \mathrm{d} \mu-\int_{Y}(q(z)-p(\alpha)) \mathrm{d} \nu \\
& =\int_{F^{+}} g(z) \mathrm{d} z-\int_{F^{-}}|g(z)| \mathrm{d} z=\int_{F} g \mathrm{~d} z
\end{aligned}
$$

Consider the optimisation problem:

$$
\begin{aligned}
& \sup J(p, q) \\
& \text { s.t. }(p, q) \in \mathcal{A}
\end{aligned}
$$

Problem (D) is a dual of problem $(\mathrm{K})$, with the weak duality property:

$$
\int_{X \times Y} c(x, y) \mathrm{d} \pi \geq J(p, q) \quad \forall \pi \in \Pi(\mu, \nu), \forall(p, q) \in \mathcal{A}
$$

This implies that problem $(\mathrm{K})$ is a also a dual to our optimum pit problem:

Proposition 5.2. $\sup (P) \leq \inf (K)$

Proof. Combining inequality (5.6) with Lemma 5.1, we get:

$$
\int_{F} g \mathrm{~d} z \leq \inf (\mathrm{K}) \quad \forall F \in \mathcal{S}(E) .
$$

In fact, by a fundamental result of Kantorovich (see [19], Thm. 1.3), there is no duality gap between (K) and (D):

$$
\inf (\mathrm{K})=\sup (\mathrm{D}) .
$$

We will show that there is also no duality gap between $(\mathrm{K})$ and $(\mathrm{P})$, i.e., that problem $(\mathrm{K})$ is a strong dual to our optimum pit problem $(\mathrm{P})$. 
Before we proceed, let us recall some facts from $c$-convex analysis. For proofs, we refer to [3], or [4]. Given $p: X \rightarrow R$ and $q: Y \rightarrow R$, we define $q^{b}: X \rightarrow R$ and $p^{\sharp}: Y \rightarrow R$ by:

$$
\begin{aligned}
& p^{\sharp}(y):=\operatorname{ess} \sup _{x \in X}(p(x)-c(x, y)), \\
& q^{b}(x):=\operatorname{ess} \inf _{y \in Y}(q(y)+c(x, y)) .
\end{aligned}
$$

Recall that $\operatorname{ess} \sup f(x)=\inf _{N \in \mathcal{N}} \sup _{x \in X \backslash N} f(x)$, where $\mathcal{N}$ is the set of measurable subsets $N \subset X$ with $\mu(N)=0$. To simplify notations, we will henceforth write sup and inf instead of ess sup and ess inf. Similarly, all equalities and inequalities have to be understood $\mu$-a.e in $X$ and $\nu$-a.e in $Y$.

It follows from the definition that:

$$
\begin{aligned}
p(x)-p^{\sharp}(y) & \leq c(x, y) \\
q^{b}(x)-q(y) & \leq c(x, y) \\
p(x) & \leq c(x, y)+p^{\sharp}(y), \text { hence } p(x) \leq p^{\sharp b}(x) \\
q(y) & \geq q^{b}(x)-c(x, y), \text { hence } q(y) \geq q^{b \sharp}(y) .
\end{aligned}
$$

We have the fundamental duality result:

$$
p^{\sharp b \sharp}=p^{\sharp} \text { and } q^{b \sharp b}=q^{b}
$$

and the monotonicity properties:

$$
\begin{aligned}
& p_{1} \leq p_{2} \Longrightarrow p_{1}^{\sharp} \leq p_{2}^{\sharp} \\
& q_{1} \leq q_{2} \Longrightarrow q_{1}^{b} \leq q_{2}^{b} .
\end{aligned}
$$

We now apply these definitions and results in our setting. Computing the right-hand sides, we get

$$
\begin{array}{ll}
p^{\sharp}(y):=\max \left\{p(\alpha), \sup _{y \in \Gamma(x)} p(x)\right\} & \text { for } y \in E^{-} \\
p^{\sharp}(\omega):=\max \left\{p(\alpha), \sup _{x \in E^{+}} p(x)-1\right\} & \\
q^{b}(x):=\min \left\{1+q(\omega), \inf _{y \in \Gamma(x)} q(y)\right\} & \text { for } x \in E^{+} \\
q^{b}(\alpha):=\min \left\{q(\omega), \inf _{y \in E^{-}} q(y)\right\} &
\end{array}
$$

with the understanding that:

$$
\sup _{x \in \varnothing} p(x)=-\infty \quad \text { and } \quad \inf _{y \in \varnothing} q(y)=+\infty .
$$

It also follows from (5.8), (5.9) and the transitivity of $\Gamma$ that:

Lemma 5.3. $p^{\sharp}$ and $q^{b}$ are increasing with respect to $\succsim_{\Gamma}$ :

$$
\begin{aligned}
& x^{\prime} \succsim_{\Gamma} x \Longrightarrow q^{b}\left(x^{\prime}\right) \geq q^{b}(x) \\
& y^{\prime} \succsim_{\Gamma} y \Longrightarrow p^{\sharp}\left(y^{\prime}\right) \geq p^{\sharp}(y) .
\end{aligned}
$$

Note that, for any given pit $F$, the associated pair $\left(p_{F}, q_{F}\right)$ defined by (5.3) and (5.4) satisfies:

$$
p_{F}=q_{F}^{b} \quad \text { and } \quad q_{F}=p_{F}^{\sharp} \text {. }
$$




\section{Solving the DUAL PROBlem}

Back to problem (D). Note that there is a built-in translation-invariance:

Lemma 6.1. Take any pair $(p, q) \in \mathcal{A}$ and any constants $p_{0}, p_{1}, q_{0}, q_{1}$ satisfying:

$$
\mu\left(E^{+}\right)\left(q_{0}-p_{1}\right)-\nu\left(E^{-}\right)\left(p_{0}-q_{1}\right)=0 .
$$

Define $(\tilde{p}, \tilde{q})$ by:

$$
\begin{aligned}
& \tilde{p}(\alpha)=p(\alpha)-p_{0} \\
& \tilde{p}(x)=p(x)-p_{1} \text { for } x \in E^{+} \\
& \tilde{q}(\omega)=q(\omega)-q_{0} \\
& \tilde{q}(y)=q(y)-q_{1} \text { for } y \in E^{-} .
\end{aligned}
$$

Then:

$$
J(\tilde{p}, \tilde{q})=J(p, q)
$$

Proof. Substituting, we get:

$$
\begin{aligned}
J(\tilde{p}, \tilde{q}) & =\int_{E^{+}}(\tilde{p}(x)-\tilde{q}(\omega)) \mathrm{d} \mu-\int_{E^{-}}(\tilde{q}(y)-\tilde{p}(\alpha)) \mathrm{d} \nu \\
& =J(p, q)+\mu\left(E^{+}\right)\left(q_{0}-p_{1}\right)-\nu\left(E^{-}\right)\left(p_{0}-q_{1}\right) .
\end{aligned}
$$

Lemma 6.2. If $(p, q) \in \mathcal{A}$, then $\left(p, p^{\sharp}\right) \in \mathcal{A},\left(q^{b}, q\right) \in \mathcal{A}$ and:

$$
\begin{gathered}
J\left(p, p^{\sharp}\right) \geq J(p, q) \\
J\left(q^{b}, q\right) \geq J(p, q) .
\end{gathered}
$$

Proof. Since $(p, q) \in \mathcal{A}$, we have $p(x)-q(y) \leq c(x, y)$ for all $(x, y)$, so that:

$$
\begin{aligned}
& p(x) \leq \inf _{y}\{c(x, y)+q(y)\}=q^{b}(x) \\
& q(y) \geq \sup _{x}\{p(x)-c(x, y)\}=p^{\sharp}(y) .
\end{aligned}
$$

Substituting into $J$, we get the result.

It follows from the Lemma that:

$$
J(p, q) \leq J\left(p, p^{\sharp}\right) \leq J\left(p^{\sharp b}, p^{\sharp}\right) .
$$

Setting $\bar{p}:=p^{\sharp b}$ and $\bar{q}:=p^{\sharp}$, we find that:

$$
\begin{aligned}
& J(p, q) \leq J(\bar{p}, \bar{q}) \\
& \bar{p}=\bar{q}^{b} \text { and } \bar{q}=\bar{p}^{\sharp} .
\end{aligned}
$$

Proposition 6.3. Problem (D) has a solution $(\bar{p}, \bar{q})$ with

$$
\begin{array}{rll}
\bar{p}=\bar{q}^{b} & \text { and } & \bar{q}=\bar{p}^{\sharp} \\
0 \leq \bar{p} \leq 1 & \text { and } & 0 \leq \bar{q} \leq 1 \\
\bar{p}(\alpha)=0 & \text { and } & \bar{q}(\omega)=0 .
\end{array}
$$


Proof. Take a maximizing sequence $\left(p_{n}, q_{n}\right) \in \mathcal{A}$ :

$$
J\left(p_{n}, q_{n}\right) \rightarrow \sup \{J(p, q) \mid(p, q) \in \mathcal{A}\} .
$$

By Lemma 6.2 and the following observations, we may assume that:

$$
p_{n}=q_{n}^{b}, \quad q_{n}=p_{n}^{\sharp} .
$$

By Lemma 6.1, we may assume in addition that:

$$
p_{n}(\alpha)=0, \quad q_{n}(\omega)=0, \quad \inf _{y \in E^{-}} q_{n}(y)=0 .
$$

If follows from (6.1) and (5.9) that, for all $x \in E^{+}$,

$$
p_{n}(x)=\min \left\{1, \inf _{y \in \Gamma(x)} q_{n}(y)\right\} .
$$

Taking (6.2) into account, we find that $0 \leq p_{n}(x) \leq 1$. Similarly, it follows from (6.1) and (5.8) that, for all $y \in E^{-}$,

$$
q_{n}(y)=\max \left\{0, \sup _{y \in \Gamma(x)} p_{n}(x)\right\} .
$$

and, since all $p_{n}(x) \leq 1$, we find that $0 \leq q_{n}(x) \leq 1$ as well.

So the family $\left(p_{n}, q_{n}\right)$ is equi-integrable in $L^{1}(\mu) \times L^{1}(\nu)$. By the Dunford-Pettis theorem, we can extract a subsequence which converges weakly to some $(p, q)$. Since the admissible set $\mathcal{A}$ is convex and closed in $L^{1}(\mu) \times L^{1}(\nu)$, it is weakly closed, and $(p, q) \in \mathcal{A}$. Since $J$ is linear and continuous on $L^{1}(\mu) \times L^{1}(\nu)$, we get:

$$
J(\bar{p}, \bar{q})=\lim _{n} J\left(p_{n}, q_{n}\right)=\sup _{\mathcal{A}} J
$$

so that $(\bar{p}, \bar{q}) \in \mathcal{A}$ is an optimal solution.

\section{Solving THE ORIGINAL PROBLEM}

We now derive the complementarity conditions arising from the strong duality equation (5.7). If $\pi$ is optimal in problem $(\mathrm{K})$ and $(p, q)$ is optimal in problem $(\mathrm{D})$, we have:

$$
\begin{aligned}
0 & =J(p, q)-\int_{X \times Y} c(x, y) \mathrm{d} \pi \\
& =\int_{X} p \mathrm{~d} \mu-\int_{Y} q \mathrm{~d} \nu-\int_{X \times Y} c(x, y) \mathrm{d} \pi \\
& =\int_{X \times Y}(p(x)-q(y)-c(x, y)) \mathrm{d} \pi .
\end{aligned}
$$

Since the integrand is non-positive and the integral is zero, the integrand must vanish almost everywhere and we obtain the complementary slackness conditions:

$$
p(x)-q(y)-c(x, y)=0 \quad \pi \text {-a.e. }
$$

Lemma 7.1. If $(p, q)$ is an optimal solution to problem $(D)$ satisfying the properties in Proposition 6.3, then we have:

$$
y^{\prime \prime} \succsim_{\Gamma} y^{\prime} \succsim_{\Gamma} x^{\prime \prime} \succsim_{\Gamma} x^{\prime} \Longrightarrow q\left(y^{\prime \prime}\right) \geq q\left(y^{\prime}\right) \geq p\left(x^{\prime \prime}\right) \geq p\left(x^{\prime}\right) .
$$


Proof. The first and the last inequality come from Lemma 5.3, and the middle one from (5.8):

$$
q\left(y^{\prime}\right)=p^{\sharp}\left(y^{\prime}\right)=\max \left\{0, \max _{x: y^{\prime} \in \Gamma(x)} p(x)\right\} \geq p\left(x^{\prime \prime}\right) .
$$

Proposition 7.2. Let $(p, q)$ be an optimal solution to problem (D) satisfying the properties in Proposition 6.3. Define the set $F \subset E$ by:

$$
F=\{x \mid p(x)=1\} \cup\{y \mid q(y)=1\} .
$$

Then $F$ is stable, and it is an optimal pit, that is, an optimal solution to problem $(P)$

Proof. Standard arguments (reduction to countable sup's and inf's) may be used to show that $F$, as defined in (7.2), is measurable. By Lemma 7.1, $F$ is stable, so it is a pit. Define $F^{+}$and $F^{-}$as in Lemma 5.1. The profit from pit $F$ satisfies:

$$
\int_{F} g(z) \mathrm{d} z=\int_{F^{+}} \mathrm{d} \mu-\int_{F^{-}} \mathrm{d} \nu \leq \sup (\mathrm{P}) .
$$

Set $G^{+}:=E^{+} \backslash F^{+}$and $G^{-}:=E^{-} \backslash F^{-}$. We have, taking into account the fact that $p=1$ on $F^{+}$and $q=1$ on $F^{-}$, together with $p(\alpha)=q(\omega)=0$ :

$$
J(p, q)=\int_{F^{+}} \mathrm{d} \mu-\int_{F^{-}} \mathrm{d} \nu+\int_{G^{+}} p \mathrm{~d} \mu-\int_{G^{-}} q \mathrm{~d} \nu .
$$

Since $\nu$ is the marginal of $\pi$ :

$$
\int_{G^{-}} q(y) \mathrm{d} \nu(y)=\int_{E^{+} \times G^{-}} q(y) \mathrm{d} \pi(x, y)
$$

Now observe that, since $c(x, y)=0$ or $+\infty$ for $(x, y) \in E^{+} \times E^{-}$, property (7.1) and the fact that $p$ and $q$ are bounded (viz., $0 \leq p \leq 1$ and $0 \leq q \leq 1$ ) imply that $p(x)=q(y) \pi$-a.e. on $E^{+} \times E^{-}$. Therefore

$$
\pi\left(F^{+} \times G^{-}\right)=0=\pi\left(G^{+} \times F^{-}\right)
$$

and thus

$$
\begin{aligned}
\int_{E^{+} \times G^{-}} q(y) \mathrm{d} \pi(x, y) & =\int_{G^{+} \times G^{-}} q(y) \mathrm{d} \pi(x, y)=\int_{G^{+} \times G^{-}} p(x) \mathrm{d} \pi(x, y) \\
& =\int_{G^{+} \times E^{-}} p(x) \mathrm{d} \pi(x, y)=\int_{G^{+}} p(x) \mathrm{d} \mu(x) .
\end{aligned}
$$

This implies:

$$
J(p, q)=\int_{F^{+}} \mathrm{d} \mu-\int_{F^{-}} \mathrm{d} \nu=\int_{F} g(z) \mathrm{d} z .
$$

Since $(p, q)$ is optimal, $J(p, q)=\sup (\mathrm{D})=\inf (\mathrm{K})$. By Proposition 5.2, $\sup (\mathrm{P}) \leq \inf (\mathrm{K})$. So:

$$
\int_{F} g(z) \mathrm{d} z=\inf (\mathrm{K}) \geq \sup (\mathrm{P})
$$

Comparing with (7.3) we see that $F$ is an optimal pit for $(\mathrm{P})$, as claimed.

The pit $F$ consists of two regions, $A:=\{p=1\}$ and $B:=\{q=1\}$. We have $g \geq 0$ on $A$, so $A$ is the profitable part of the pit, while $g \leq 0$ on $B$, so $B$ is the costly part, which must be excavated in order to reach $A$. Note that $A$ need not be equal to the whole $E^{+}$: there are regions underground which are potentially profitable, but which are too costly to reach. 
Summarizing our results:

Theorem 7.3. If $E$ is compact, $\succsim_{\Gamma}$ is a preorder on $E$ with closed graph, and $g(x)$ is continuous with $\int_{E} \max \{0, g(x)\} \mathrm{d} x>0$, then:

(1) Problem $(P)$ has an optimum solution, i.e., there exists an optimal pit $F$.

(2) The corresponding pair $(p, q):=\left(p_{F}, q_{F}\right)$ defined by (5.3)-(5.4) is an optimum solution to problem $(D)$.

(3) Problem $(K)$ has an optimum solution and is a strong dual to problem $(P)$, i.e., $\min (K)=\max (P)$.

(4) A pit $F$ is optimal if and only if there exists a feasible solution $\pi$ to problem $(K)$ such that the pair $(p, q):=\left(p_{F}, q_{F}\right)$ satisfies the complementary slackness conditions (7.1).

Proof. By Proposition 6.3, there is an optimal solution $(\bar{p}, \bar{q})$ to problem (D), and by Proposition 7.2 we have $J(\bar{p}, \bar{q})=\int_{F} g(z) \mathrm{d} z=\sup (\mathrm{D})$, so the pit $F$ defined by (7.2) is optimal. On the other hand, by Lemma 5.1, we have $J\left(p_{F}, q_{F}\right)=\int_{F} g(z) \mathrm{d} z$, so the pair $\left(p_{F}, q_{F}\right) \in \mathcal{A}$ is optimal as well. The other statements follow from preceding observations.

The optimum pit need not be unique. In fact it is known (Topkis [18]; see also Matheron [12], Thm. 2) that the set of optimal pits is closed under (arbitrary) intersections and unions. Therefore, taking the intersection and the union, respectively, of all optimum pits, we have:

Corollary 7.4. There exist a unique smallest optimum pit and a unique largest optimum pit.

The smallest optimum pit may be of particular interest when seeking to minimize the environmental impact of the pit without sacrificing its total profit.

Of course, our solution of the problem is purely static: all the excavation and extraction is done at once. In practice, these processes take time, and it is of interest to plan the whole mining process so as to optimize discounted revenue over time. This leads to a variant of the optimal transportation problem, where transportation is costly, not only in money but also in time. We hope to investigate it in the not-too-distant future.

Acknowledgements. Both authors thank the Centro de Modelamiento Matematico (CMM) at the Universidad de Chile (Santiago) for its hospitality, and Professors Felipe Alvarez and Jorge Amaya for substantial discussions. ${ }^{7}$

\section{REFERENCES}

[1] F. Alvarez, A. Jorge G. Andreas and S. Nikolai, A continuous framework for open pit mine planning. Math. Methods Oper. Res. 73 (2011) 29-54

[2] D. Bienstock and Z. Mark, Solving LP Relaxations of Large-Scale Precedence constrained problems. Proc. of 14 th Conference on Integer Programming and Combinatorial Optimization (IPCO 2010). Vol. 6080 of Lect. Note Comput. Sci. Springer (2010) $1-14$.

[3] G. Carlier, Duality and Existence for a Class of Mass Transportation Problems and Economic Applications, in Adv. Math. Econ. Springer, Japan (2003) 1-21

[4] I. Ekeland, Existence, uniqueness and efficiency of equilibrium in hedonic markets with multidimensional types. Econ. Theory $42(2010) 275-315$

[5] D. Espinoza, M. Goycoolea, E. Moreno and A.N. Newman, MineLib: A library of open pit mining problems. Ann. Oper. Res. 206 (2012) 91-114

[6] A. Griewank and S. Nikolai, Duality results for stationary problems of open pit mine planning in a continuous function framework. Comput. Appl. Math. 30 (2011) 197-215.

[7] J. Guzmán, Ultimate Pit Limit Determination: A New Formulation for an Old (and Poorly Specified) Problem Workshop on Operations Research in Mining, Viña del Mar, Chile (2008) 10-12.

[8] P. Huttagosol and R.E. Cameron, A Computer Design of Ultimate Pit Limit by Using Transportation Algorithm, in Proc. of the 23rd International Symposium on Applications of Computers in Mining (1992) 443-460.

\footnotetext{
${ }^{7}$ The authors would also like to thank Professor Marcos Goycoolea for providing a copy of reference [8], and an anonymous referee for constructive suggestions that helped improve this paper.
} 
[9] Th. B. Johnson, Optimum open pit mine production scheduling. Report ORC-68-11, Operations Research Center. University of California Berkeley (1968).

[10] R. Khalokakaie, Computer-aided optimal open pit design with variable slope angles. Ph.D. thesis, University of Leeds (1999).

[11] R. Khalokakaie, P.A. Dowd and R.J. Fowell, Lerchs-Grossmann algorithm with variable slope angles. Mining Technology 109 (2000) 77-85.

[12] G. Matheron, Paramétrage de contours optimaux. Note géostatistique 128. Fontainebleau. Février (1975).

[13] G. Matheron, Compléments sur le paramétrage de contours optimaux. Note géostatistique 129. Fontainebleau, Février (1975).

[14] N. Morales, Modelos Matemáticos Para Planificación Minera. Engineering thesis. Universidad de Chile, Santiago (2002).

[15] A.M. Newman, E. Rubio, R. Caro, A. Weintraub and K. Eurek, A review of operations research in mine planning. Interfaces 40 (2010) 222-245.

[16] J.-C. Picard, Maximal closure of a graph and applications to combinatorial problems. Manag. Sci. 22 (1976) $1268-1272$

[17] Strogies, Nikolai, and Andreas Griewank, A PDE constraint formulation of Open Pit Mine Planning Problems. Proc. Appl. Math. Mech. 13 (2013) 391-392

[18] D.M. Topkis, Minimizing a submodular function on a lattice. Oper. Res. 26 (1978) 305-321.

[19] C. Villani, Topics in Optimal Transportation. In vol. 58 of Grad. Stud. Math. AMS (2003). 